\title{
A computational viscoelasticity/damage/plasticity model for high-explosive materials
}

\author{
P. Rangaswamy \& R. M. Hackett \\ Los Alamos National Laboratory, Los Alamos, New Mexico, USA
}

\begin{abstract}
It is important to be able to accurately predict the behavior of high-explosive (HE) materials because of the sensitivity with which they respond in the environment in which they are designed to perform, and because they are also extremely sensitive to accidental mechanical shock, which might occur during handling. Thus it is imperative that a capability exists to develop a constitutive material model and simulate the response of HE materials subjected to a variety of thermomechanical loading conditions, a response that is characterized as highly complex. It has been demonstrated through uniaxial strain-rate experiments conducted at the Los Alamos National Laboratory (LANL) that the initial response of HE material is rate-dependent, with the growth of microcracks continuing under load until the yield strength is reached. In a material such as this, the initial stages of deformation involve both elastic and viscous mechanisms. It has been postulated that this observed behavior could be categorized as nonlinear viscoelasticity, coupled with continuing microcracking. A computational material model containing viscoelasticity, internal damage accumulation and rate-dependent plasticity has been formulated for the purpose of simulating the behavior of these types of material systems. The formulation has been incorporated as a user-defined material (UMAT) into the ABAQUS finite element code. It is demonstrated in this paper how the material model can be employed by calibrating it to the experimental response of the material Mock 900-21, a high-explosive material mock utilized at LANL.
\end{abstract}

Keywords: computational, viscoelasticity, damage, fracture mechanics, plasticity, user material model. 


\section{Introduction}

It is important to be able to accurately predict the behavior of HE materials because of the sensitivity with which they respond in the environment in which they are designed to perform, and because they are also extremely sensitive to accidental mechanical shock, which might occur during handling. Thus it is imperative that a capability exist to model the response of $\mathrm{HE}$ materials subjected to a variety of thermo mechanical loading conditions, a response that is highly complex, requiring sophisticated material modeling to simulate.

It has been demonstrated through uniaxial strain-rate testing conducted at LANL that the initial response of HE material, along with the yield strength, is rate-dependent, with the growth of microcracks continuing under load until the yield strength is reached. In a material such as this, the initial stages of deformation involve both elastic and viscous mechanisms and are truly viscoelastic. It has been postulated that this observed behavior could be categorized as nonlinear viscoelasticity, coupled with continuing microcracking. However, since it is extremely difficult to obtain experimental data to support current nonlinear viscoelasticity theory, and since internal damage accumulation modeling has been successfully employed in numerous circumstances in lieu of nonlinear viscoelasticity modeling [1], a linear viscoelasticity/damage approach has been taken here in modeling the behavior of the HE material. Also, since certain HE materials have been observed to exhibit flow characteristics subsequent to reaching the damage-controlled yield stress, an applicable plasticity theory is presented and developed.

The theory presented herein is used to develop a UMAT subroutine for the ABAQUS implicit finite element code. Mock materials are developed to simulate the laboratory response of HE materials because the mock materials can be conventionally tested whereas the HE materials usually cannot be. The developed UMAT is calibrated for Mock 900-21, a mock material for the highexplosive material PBX 9501, and uniaxial tests performed on Mock 900-21 are simulated. The calibrations are described in detail and simulation results are compared with test results.

\section{Model formulation}

The time dependence of microcrack growth in polymeric materials has been studied extensively, both theoretically and experimentally, for quite some time. The linear viscoelasticity/damage formulation presented here is based primarily upon the work of Addessio and Johnson [2]. During deformation, the distribution of microcracks in the material is assumed to be random, with the initial distribution being exponential.

\subsection{Linear viscoelasticity/damage}

Using Cartesian tensor index notation, strain is given by the kinematic relationship 


$$
\varepsilon_{i j}=\frac{1}{2}\left(u_{j, i}+u_{i, j}\right), \quad i, j=1,2,3
$$

where the $u_{i}$ are the material deformation variables. It can be decomposed into deviatoric and mean components

$$
\varepsilon_{i j}=e_{i j}+\varepsilon_{m} \delta_{i j}
$$

where $\delta_{i j}$ is the Kronecker delta; the mean strain is defined by

$$
\varepsilon_{m}=\frac{1}{3} \varepsilon_{i i}
$$

The stress can likewise be decomposed into deviatoric and mean components

$$
\sigma_{i j}=s_{i j}+\sigma_{m} \delta_{i j}
$$

where the mean stress is defined by

$$
\sigma_{m}=\frac{1}{3} \sigma_{i i}
$$

and is related to the mean strain through the expression

$$
\sigma_{m}=3 K \varepsilon_{m}
$$

where $K$ is the bulk modulus of the material.

In a deviatoric Maxwell model, a single spring and dashpot in series, the relationship between stress and strain is

$$
s_{i j}=2 G e_{i j}^{e}
$$

where $\mathrm{G}$ is the shear modulus, and the relationship between stress and viscous strain rate is

$$
s_{i j}=2 \eta \dot{e}_{i j}^{v}
$$

where $\eta$ is the viscosity of the dashpot and where the dot over the variable indicates the time rate of change of the variable. Taking the partial derivative of Equation (7) and combining the result with Equation (8), i.e. adding elastic and viscous strain rates, gives

$$
\dot{s}_{i j}=2 G \dot{e}_{i j}^{v e}-\frac{s_{i j}}{\tau}
$$

where the relaxation time $\tau$ is given by

$$
\tau=\frac{\eta}{G}
$$

For the viscoelastic solid, represented by a generalized deviatoric Maxwell model, with the strain being common for all elements of the model and the stresses for the individual elements being additive, i.e.

$$
S_{i j}=\sum_{n=1}^{N} S_{i j}^{(n)}
$$


where $N$ is the number of elements in the generalized Maxwell model and $S_{i j}^{(n)}$ is the deviatoric stress component for the $n$th element, the relationship between the deviatoric stress rate and the viscoelastic deviatoric strain rate and the deviatoric stress, referring to Equation (9), is given by

$$
\dot{s}_{i j}=\sum_{n=1}^{N}\left(2 G^{(n)} \dot{e}_{i j}^{v e}-\frac{s_{i j}^{(n)}}{\tau^{(n)}}\right)
$$

where $G^{(n)}$ and $\tau^{(n)}$ are the shear modulus and relaxation time, respectively, for the $n$th Maxwell element.

The cracking deviatoric strain versus deviatoric stress relationship, see Addessio and Johnson [2], is

$$
e_{i j}^{c r}=\beta c^{3} s_{i j}
$$

where $\mathrm{c}$ is the value of the average microcrack radius and $\beta$ is a parameter that relates the shear modulus to the initial flaw size through the expression

$$
\beta=\frac{1}{2 G a^{3}}
$$

where $a$ is the limiting crack size and

$$
G=\sum_{n=1}^{N} G^{(n)}
$$

Combining Equations (13) and (14) yields a relationship between the deviatoric strain and the average crack radius

$$
2 G e_{i j}^{c r}=\left(\frac{c}{a}\right)^{3} s_{i j}
$$

Taking the partial derivative of Equation (16) with respect to time gives the expression

$$
2 G \dot{e}_{i j}^{c r}=3\left(\frac{c}{a}\right)^{2} \frac{c}{a} s_{i j}+\left(\frac{c}{a}\right)^{3} \dot{s}_{i j}
$$

The total deviatoric strain, the sum of the viscoelastic deviatoric strain and the cracking deviatoric strain, is given by

$$
e_{i j}=e_{i j}^{v e}+e_{i j}^{c r}
$$

Combining Equations (12), (17) and (18) gives an expression for the deviatoric stress in terms of combined viscoelastic and microcracking response:

$$
\dot{s}_{i j}=\psi \dot{e}_{i j}-\theta\left(s_{i j}+\lambda_{i j}\right)
$$

where

$$
\begin{gathered}
\psi=\frac{2 G}{1+(c / a)^{3}} \\
\theta=\frac{3(c / a)^{2}(\dot{c} / a)}{1+(c / a)^{3}}
\end{gathered}
$$

and 


$$
\lambda_{i j}=\frac{\sum_{n=1}^{N} s_{i j}^{(n)} / \tau^{(n)}}{3(c / a)^{2}(\dot{c} / a)}
$$

The expression for the deviatoric stress rate for the $n$th Maxwell element is given by

$$
\dot{s}_{i j}^{(n)}=2 G^{(n)} \dot{e}_{i j}-\frac{s_{i j}^{(n)}}{\tau^{(n)}}-\frac{G^{(n)}}{G}\left[3\left(\frac{c}{a}\right)^{2} \frac{\dot{c}}{a} s_{i j}+\left(\frac{c}{a}\right)^{3} \dot{s}_{i j}\right]
$$

\subsection{Fracture mechanics}

The application of fracture mechanics to time-dependent materials is relatively new [3]. An evolution equation defining crack growth rate is required. Consistent with traditional fracture-mechanics reasoning, and following the observations and conclusions of Dienes [4], it is assumed that the growth rate of the average crack radius is functionally dependent upon the stress intensity. The equations below are from Dienes [5] and Dienes and Kershner [6], with modifications for tension and friction provided by Johnson [7]. The cracking rate is either

$$
\dot{c}=v_{\text {res }}\left(\frac{K_{I}}{K_{1}}\right)^{m}, \quad K_{I}<K^{\prime}
$$

or

$$
\dot{c}=v_{\text {res }}\left[1-\left(\frac{K_{0 \mu}}{K_{I}}\right)^{2}\right], \quad K_{I} \geq K^{\prime}
$$

where

$$
K_{I}=\left(\frac{3 \pi c}{2} s_{i j} s_{i j}\right)^{1 / 2}, \quad \sigma_{m}<0 \quad \text { (compression) }
$$

or

$$
K_{I}=\left(\frac{3 \pi c}{2} \sigma_{i j} \sigma_{i j}\right)^{1 / 2}, \quad \sigma_{m} \geq 0 \quad \text { (tension) }
$$

and

$$
\begin{gathered}
K^{\prime}=K_{0 \mu}\left(1+\frac{2}{m}\right)^{1 / 2} \\
K_{1}=K^{\prime}\left(1+\frac{m}{2}\right)^{1 / m} \\
K_{0 \mu}=K_{0}\left[1-\frac{\pi \mu^{\prime} \sigma_{m} c^{1 / 2}}{K_{0}}\left(1-\frac{\mu^{\prime} \sigma_{m} c^{1 / 2}}{K_{0}}\right)\right]^{1 / 2} \\
\mu^{\prime}=\left[\frac{45}{2\left(3-2 \mu_{s}^{2}\right)}\right]^{1 / 2} \mu_{s}
\end{gathered}
$$


where $v_{\text {res }}$ is the value of the rate of growth of the average crack radius $K_{\mathrm{o}}$ is the threshold value of stress intensity, $m$ is a cracking parameter and $\mu_{\mathrm{s}}$ is the static coefficient of friction.

For the $N$-component Maxwell model, the viscous work rate is

$$
\dot{w}^{v e}=\sum_{n=1}^{N} s_{i j}^{(n)} \dot{e}_{i j}^{v e}=\sum_{n=1}^{N} \frac{S_{i j}^{(n)} S_{i j}^{(n)}}{2 G^{(n)} \tau^{(n)}}
$$

and the cracking damage work rate is

$$
\dot{w}^{c r}=s_{i j} \dot{e}_{i j}^{c r}=\frac{1}{2 G}\left[3\left(\frac{c}{a}\right)^{2} \frac{\dot{c}}{a} s_{i j} s_{i j}+\left(\frac{c}{a}\right)^{3} s_{i j} \dot{s}_{i j}\right]
$$

Employing the constitutive model formulation presented, the incremental form of the equations, facilitating a computational solution, will be developed. Combining Equations (4), (6) and (19) yields the expression

$$
\theta \sigma_{i j}+\dot{\sigma}_{i j}=3 K\left(\theta \varepsilon_{m}+\dot{\varepsilon}_{m}\right)+\psi \dot{e}_{i j}-\theta \lambda_{i j}
$$

Rearranging Equation (34) somewhat yields the expression

$$
\theta \sigma_{x x}+\dot{\sigma}_{x x}=\theta K \varepsilon_{V}+\left(K-\frac{\psi}{3}\right) \dot{\varepsilon}_{V}+\psi \dot{\varepsilon}_{x x}-\theta \lambda_{x x}
$$

for the global $\mathrm{x}$-direction on a plane $\mathrm{x}=$ constant, with similar expressions for the $\mathrm{y}$ - and the $\mathrm{z}$-direction, and

$$
\theta \sigma_{x y}+\dot{\sigma}_{x y}=\frac{\psi}{2} \dot{\varepsilon}_{x y}-\theta \lambda_{x y}
$$

for the $\mathrm{x}$-direction on the plane $\mathrm{y}=$ constant, with similar expressions for the $\mathrm{y}$ and the z-direction, where

$$
\varepsilon_{V}=\varepsilon_{i i}
$$

The factor of 2 in the denominator in Equation (36) indicates that the strains are engineering.

Applying the central difference operator to Equations (35) and (36) yields

$$
\left(1+\frac{\theta \Delta t}{2}\right) \Delta \sigma_{x x}=\left[K\left(1+\frac{\theta \Delta t}{2}\right)-\frac{\psi}{3}\right] \Delta \varepsilon_{V}+\psi \Delta \varepsilon_{x x}+\theta \Delta t\left(K \varepsilon_{V}-\sigma_{x x}-\lambda_{x x}\right)_{t}
$$

and

$$
\left(1+\frac{\theta \Delta t}{2}\right) \Delta \sigma_{x y}=\frac{\psi}{2} \Delta \varepsilon_{x y}-\theta \Delta t\left(\sigma_{x y}+\lambda_{x y}\right)_{t}
$$

where $\Delta$ indicates incremental change, so that the Jacobian matrix $D$ then has the terms

$$
\begin{gathered}
D(1,1)=\frac{\partial \Delta \sigma_{x x}}{\partial \Delta \varepsilon_{x x}}=K+\frac{2 \psi}{3(1+\theta \Delta t / 2)} \\
D(1,2)=\frac{\partial \Delta \sigma_{x x}}{\partial \Delta \varepsilon_{y y}}=K-\frac{\psi}{3(1+\theta \Delta t / 2)}, \text { etc. }
\end{gathered}
$$

and 


$$
D(4,4)=\frac{\partial \Delta \sigma_{x y}}{\partial \Delta \sigma_{x y}}=\frac{\psi}{2(1+\theta \Delta t / 2)}, \text { etc. }
$$

crack growth. In a simulation of a uniaxial strain-rate test, the value of $v_{\text {res }}$ is changed until the yield stress of the simulation matches that of an actual test run at the same strain-rate. This procedure is repeated for a test run at a different rate of strain until a wide range of strain-rate tests have been covered. This results in a collection of say five data points, each of which relates a value of $v_{\text {res }}$ to a given value of strain-rate. A log-log plot of these data can usually be expected to yield a linear relationship between $\log \left(v_{\text {res }}\right)$ and the log of the effective strain rate (for three-dimensionality) where the effective strain rate is given by

$$
\dot{\varepsilon}_{e f f}=\frac{\sqrt{2}}{2(1+v)}\left[\left(\dot{\varepsilon}_{x x}-\dot{\varepsilon}_{y y}\right)^{2}+\left(\dot{\varepsilon}_{y y}-\dot{\varepsilon}_{z z}\right)^{2}+\left(\dot{\varepsilon}_{z z}-\dot{\varepsilon}_{x x}\right)^{2}+\frac{3}{2}\left(\dot{\varepsilon}_{x y}^{2}+\dot{\varepsilon}_{y z}^{2}+\dot{\varepsilon}_{z x}^{2}\right)\right]^{1 / 2}
$$

where the strains are engineering and where $v$, Poisson's ratio, is given by

$$
v=\frac{K-\frac{\psi}{3(1+\theta \Delta t / 2)}}{2 K+\frac{\psi}{3(1+\theta \Delta t / 2)}}
$$

\subsection{Plasticity}

Plasticity theory adaptable to computational modeling is generally well developed $[8,9]$. An example of combining damage growth and plasticity is reported by Khaleel et al. [10], and the coupling of yield surface to damage is discussed by Johansson and Runesson [11]. The yield condition modeled herein is basically the flow rule of von Mises' theory, the well-known Prandtl-Reuss relationship [12]. The effective plastic strain increment $\Delta \varepsilon_{\text {eff }}^{p}$ is defined by

$$
\begin{gathered}
\lfloor W\rfloor=\frac{\{Q\}^{T}[D]}{\left(H+\{Q\}^{T}[D]\{Q\}\right)} \\
\{Q\}=\frac{3}{\sigma_{e f f}}\left\{\frac{s_{x x}}{2}, \frac{s_{y y}}{2}, \frac{s_{z z}}{2}, s_{x y}, s_{y z}, s_{z x}\right\} \\
\sigma_{e f f}=\frac{\sqrt{2}}{2}\left[\left(\sigma_{x x}-\sigma_{y y}\right)^{2}+\left(\sigma_{y y}-\sigma_{z z}\right)^{2}+\left(\sigma_{z z}-\sigma_{x x}\right)^{2}+6\left(\sigma_{x y}^{2}+\sigma_{y z}^{2}+\sigma_{z x}^{2}\right)\right]^{1 / 2}
\end{gathered}
$$

and where $H$ is the hardening (or softening) modulus. The Prandtl-Reuss relationship states that plastic strain increments $\left\{\Delta \varepsilon^{p}\right\}$ result when the effective plastic strain increment occurs under the state of stress $\{Q\}$, i.e.

$$
\left\{\Delta \varepsilon^{p}\right\}=\{Q\} \Delta \varepsilon_{\text {eff }}^{p}
$$

The corresponding stress increments are obtained from the relationship 


$$
\{\Delta \sigma\}=[D]\left(\{\Delta \varepsilon\}-\left\{\Delta \varepsilon^{p}\right\}\right)
$$

where

$$
\{\Delta \sigma\}=\left\{\Delta \sigma_{x x}, \Delta \sigma_{y y}, \Delta \sigma_{z z}, \Delta \sigma_{x y}, \Delta \sigma_{y z}, \Delta \sigma_{z x}\right\}
$$

It is assumed that, consistent with plasticity theory in general, the hardening modulus depends on the strain and the strain rate [13]. The dependence upon strain rate is expressed as a shift function with regard to a basis value of strain rate. It has the form

$$
a_{S}=\frac{s_{1}\left(\dot{\varepsilon}_{e f f}-\dot{\varepsilon}_{e f f}^{0}\right)}{s_{2}+\left(\dot{\varepsilon}_{e f f}-\dot{\varepsilon}_{e f f}^{0}\right)}
$$

where $\dot{\varepsilon}_{\text {eff }}^{0}$ is the basis value of strain rate and $s_{1}$ and $s_{2}$ are constants to be determined from curve fitting. The dependence of the hardening modulus on strain is expressed as

$$
H=a_{1}+a_{2}\left(\varepsilon_{e f f}-a_{S}\right)+a_{3}\left(\varepsilon_{e f f}-a_{S}\right)^{2}
$$

where

$$
\varepsilon_{e f f}=\frac{\sqrt{2}}{2(1+\nu)}\left[\left(\varepsilon_{x x}-\varepsilon_{y y}\right)^{2}+\left(\varepsilon_{y y}-\varepsilon_{z z}\right)^{2}+\left(\varepsilon_{z z}-\varepsilon_{x x}\right)^{2}+\frac{3}{2}\left(\varepsilon_{x y}^{2}+\varepsilon_{y z}^{2}+\varepsilon_{z x}^{2}\right)\right]^{1 / 2}
$$
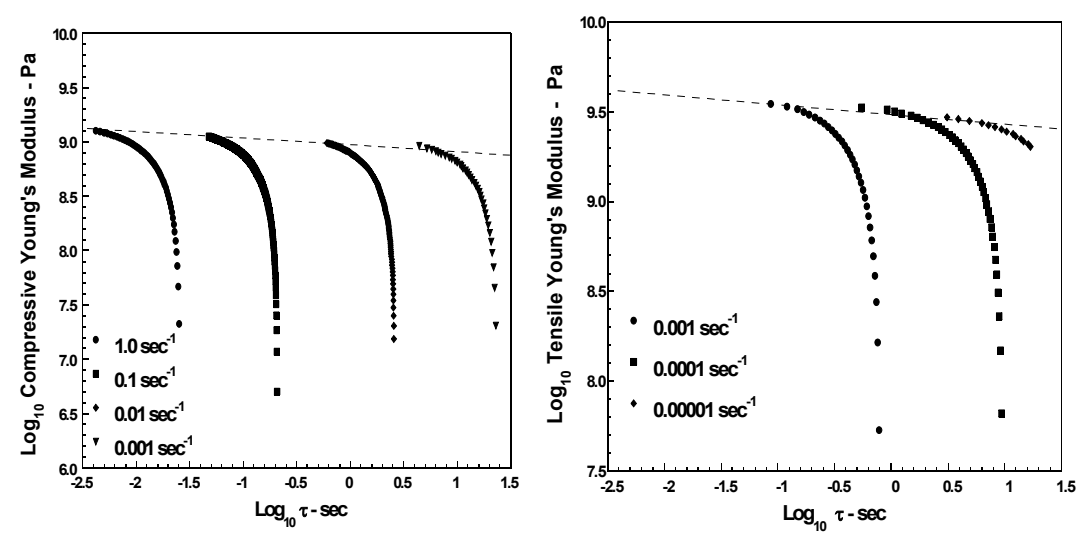

Figure 1: Log compressive (left) and tensile (right) Young's modulus versus Log relaxation time for Mock 900-21.

\section{Application}

The formulation thus presented has been used to develop a User MATerial Subroutine (UMAT) for the ABAQUS implicit finite element code. Mock materials are developed to simulate the response of HE materials because the mock materials can be conventionally tested whereas the HE materials usually cannot be. For example, Mock 900-21 is designed to simulate the strength and 
density properties of the high-explosive material PBX 9501. The theory/formulation described herein is applied to Mock 900-21 for the purpose of validating the material model.
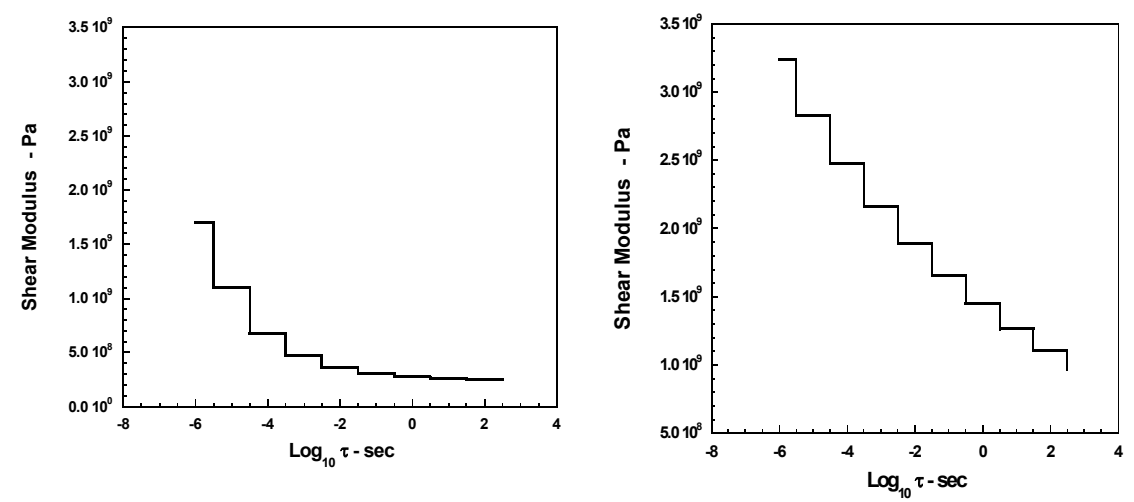

Figure 2: Log compressive (left) and tensile (right) Young's modulus versus Log relaxation time for Mock 900-21.
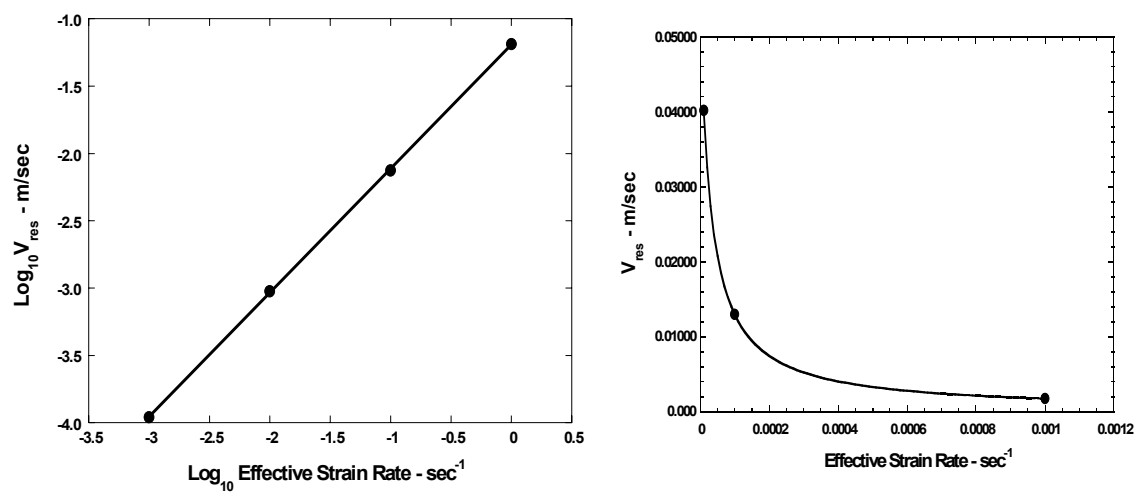

Figure 3: Relationship between log of rate of growth of crack radius and log of effective strain rate in compression (left) and tension (right) for Mock 900-21.

Compressive strain rate data were obtained from tests conducted by Cady [14], and tensile strain-rate test data were obtained from tests conducted by Thompson [15]. Compressive tests were for rates of $0.001,0.01,0.1$ and 1.0 per second. Tensile tests were for rates of $0.00001,0.0001$ and 0.001 per second. These tests were used exclusively to determine the viscoelastic, damage and plasticity parameters employed in the material model to predict the response of the Mock 900-21. Each set of uniaxial strain-rate data was converted from a true stress versus true strain data set to a Young's modulus $(\mathrm{Pa})$ versus relaxation 
time (sec) data set. The relaxation time was obtained by dividing strain by strain rate. Each of these data sets was then converted to a log Young's modulus (Pa) versus $\log \tau$ (sec) data set. These data are shown plotted in Figure 1. Fitting the upper-most data points in Figure 1 yields expressions for the compressive relaxation modulus and the tensile relaxation modulus, respectively.

With a value of Poisson's ratio of 0.30 , and 10 generalized deviatoric Maxwell model elements, each separated by a decade of relaxation time, as shown in Figure 2, the linear viscoelastic models for the material are established.

It was found, and is thus noted, that the elastic/viscoelastic material properties for Mock 900-21 are different in compression and tension. For the compressive strain-rate tests, using the procedure described earlier, a linear relationship between $\log \left(v_{\text {res })}\right)$ and $\log \left(\dot{\varepsilon}_{\text {eff }}\right)$ was established for Mock 900-21. This is shown in Figure 3. The yield behavior of Mock 900-21 in compression and in tension is also quite different, as one might expect. For tensile strain-rate tests, again using the procedure described earlier, a relationship between $v_{\text {res }}$ and $\dot{\varepsilon}_{\text {eff }}$ was established; this relationship is shown in Figure3. The numerical values of the plasticity parameters found in Equations (51) were $s_{1}=-4.0322 \times 10^{-5}$, $s_{2}=1.1178 \times 10^{-3} \mathrm{sec}^{-1}$, and $\dot{\varepsilon}_{\text {eff }}^{0}=0.001 \mathrm{sec}^{-1}$, and the values of those found in Equations (54) were $a_{1}=4.7863 \times 10^{3} \quad \mathrm{~Pa}, \quad a_{2}=6.1878 \times 10^{6} \quad \mathrm{~Pa}, \quad$ and $a_{3}=2.1976 \times 10^{9} \mathrm{~Pa}$.

Finally, comparisons between model predictions and test results are shown in Figures 4 and 5, Figure 4 for compressive loading and Figure 5 for tensile loading.

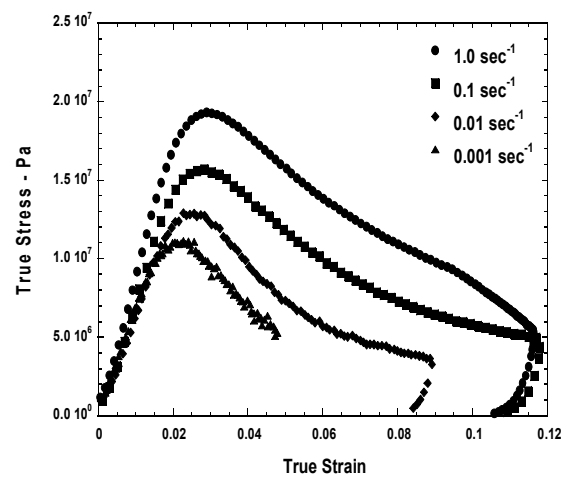

(a)

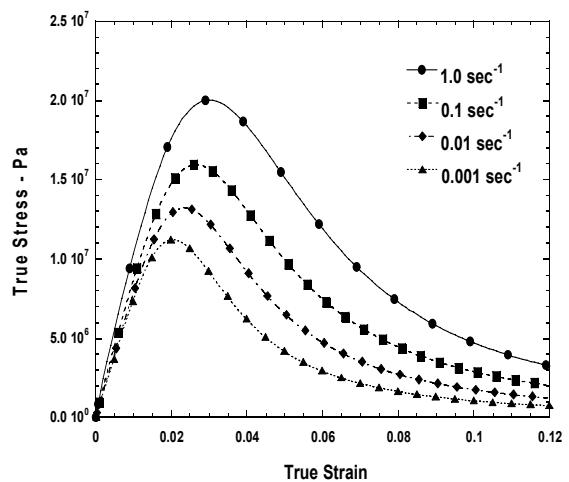

(b)

Figure 4: Uniaxial compressive stress-strain relationship for Mock 900-21:

(a) test data, (b) simulation. 


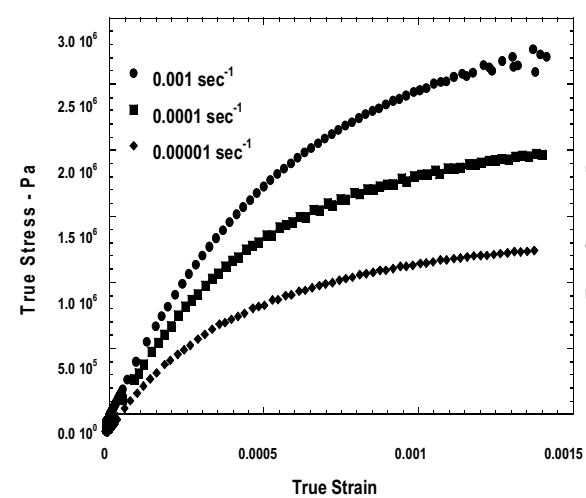

(a)

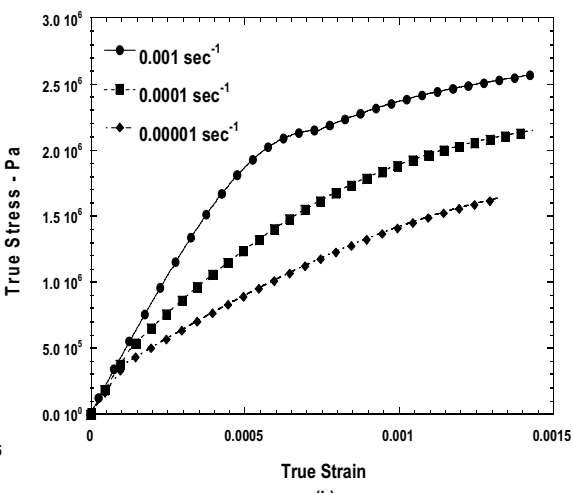

(b)

Figure 5: Uniaxial tensile stress-strain relationship for Mock 900-21: (a) test data, (b) simulation.

\section{Conclusions}

Results obtained from the analyses performed using this material model for the Mock 900-21material indicates that the model performs satisfactorily. Since Mock 900-21 exhibits flow characteristics subsequent to reaching its damagecontrolled yield strength in tension, the plasticity theory developed for computational modeling in conjunction with the coupled viscoelastic/damage model was applicable to this material.

\section{References}

[1] Schapery, R.A., Nonlinear Viscoelastic Solids, International Journal of Solids and Structures, 37, pp 359-366, 2000.

[2] Addessio, F.L. \& Johnson, J.N., A Constitutive Model for the Dynamic Response of Brittle Materials, Journal of Applied Physics, 67(7), pp. 3275-3286, 1990.

[3] Anderson, T.L., Fracture Mechanics, Fundamentals and Applications, Second Edition, CRC Press, Inc., pp. 31-99, 205-220, 1995.

[4] Dienes, J.K., A Unified Theory of Flow, Hot-Spots, and Fragmentation with an Application to Explosive Sensitivity. High-Pressure Shock Compression of Solids II, eds. L. Davison, D.E. Grady \& M. Shahinpoor, Springer Verlag: New York, Chapter 14, pp. 366-398, 1996.

[5] Dienes, J.K., Strain-Softening via SCRAM, Los Alamos National Laboratory Report, LA-UR-98-3620, 1998.

[6] Dienes, J.K. and Kershner, J.D., Multiple-Shock Initiation via Statistical Crack Mechanics, Proceedings of the $11^{\text {th }}$ Symposium on Detonation, eds. J.M. Short \& J.E. Kennedy, Snowmass, Colorado, USA, 1998.

[7] Johnson, J.N. Personal communication, May 2004, Technical Staff Member, Los Alamos National Laboratory, Los Alamos, USA. 
[8] Lubliner, J. Plasticity Theory, Macmillan Publishing Company, 1990

[9] Simo, J.C. \& Hughes T.J.R., Computational Inelasticity, Springer-Verlag New York, 1998.

[10] Khaleel, M.A., Zbib, H.M., \& Nyberg, E.A., 2001, Constitutive Modeling of Deformation and Damage in Superplastic Materials, International Journal of Plasticity, 17, 277-296.

[11] Johansson, M. \& K. Runesson, K., Viscoplasticity with Dynamic Yield Surface Coupled to Damage, Computational Mechanics, 20, pp 53-59, 1997.

[12] Steeb, H. and E. Ramm, A General Framework for Local Error Estimation Applied to Material Nonlinear Problems, European Congress on Computational Methods in Applied Sciences and Engineering, ECCOMAS 2000, Barcelona, 11-14 September, p. 1-14, 2000.

[13] Fish, J. \& Shek, K., Computational Plasticity and Viscoplasticity for Composite Materials and Structures, Composites Part B, 29B, pp613-619, 1998.

[14] Cady, C.M. Personal communication, Aug 2004, Technical Staff Member, Los Alamos National Laboratory, Los Alamos, USA.

[15] Thompson, D.G., Personal communication, Aug 2004, Technical Staff Member, Los Alamos National Laboratory, Los Alamos, USA. 